\title{
A design of experiment rational optimization of the degumming process and its impact on the silk fibroin properties.
}

Alessio Bucciarelli ${ }^{*}$, Gabriele Greco ${ }^{4}$, Ilaria Corridori ${ }^{4}$, Nicola M. Pugno ${ }^{4,5}$, Antonella Motta ${ }^{2,3}$

${ }^{1}$ Microsystem Technology Group, Center for materials and microsystems, Fondazione Bruno Kessler, Via Sommarive 9, 38123 Trento (TN), Italy

${ }^{2}$ Department of Industrial Engieneering, University of Trento, via Delle Regole 101, Trento 38123, Italy

${ }^{3}$ BIOTech Research Center and European Institute of Excellence on Tissue Engineering and Regenerative Medicine, Via delle Regole 101, Trento 38123, Italy

${ }^{4}$ Laboratory of Bio-inspired, Bionic, Nano, Meta Materials and Mechanics, Department of Civil, Environmental and Mechanical Engineering, University of Trento, Via Mesiano 77, 38123 Trento (TN), Italy

${ }^{5}$ School of Engineering and Materials Science, Queen Mary University of London, Mile End Road, E14NS London, United Kingdom 


\section{Calibration Curves}

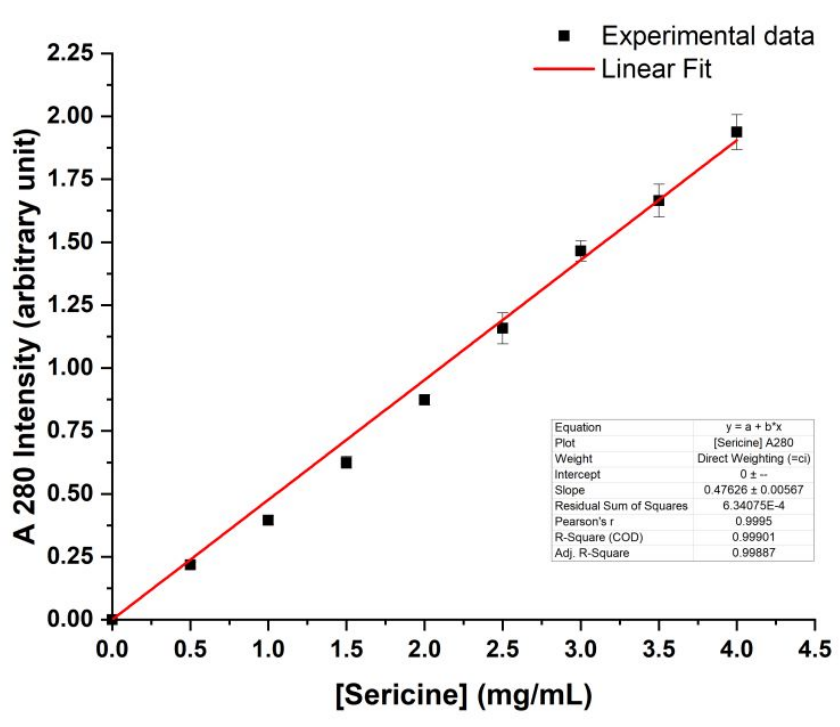

Figure S 1: Calibration curve used to determine the concentration of sericin in the degumming baths. The curve was prepared by standard solutions with a known concentration of sericin versus the intensity at $280 \mathrm{~nm}$ of their UV/VIS spectra. This curve was used to determine the unknown concentration of sericin the degumming bath by evaluating the UV intensity at $280 \mathrm{~nm}$ and interpolating the corresponding sericin concentration.

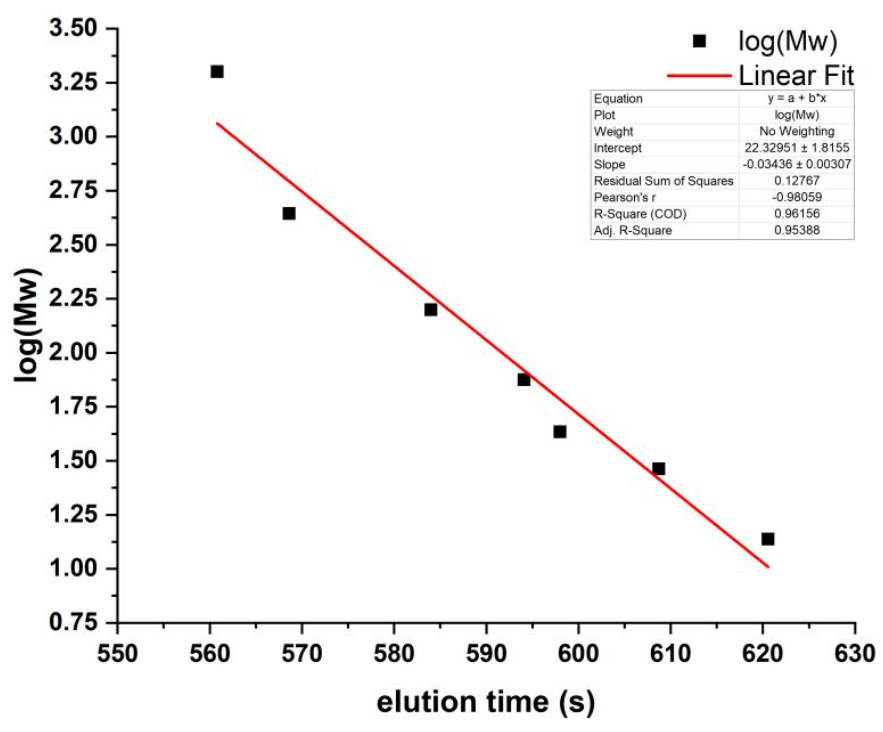

Figure S 2: Calibration curve of the molecular weight analysis conducted by Gel Permeation Chromatography (GPC). The curve was produced by using reference proteins with a known molecular weight. 


\section{FTIR Assignment}

\begin{tabular}{c|c|c} 
Assignment & $\begin{array}{c}\text { Peak position } \\
\text { cm}^{-1}\end{array}$ & Reference \\
\hline Side chain & $1597-1609$ & $1-4$ \\
Antiparallel $\beta$ - sheet & $1610-1625$ & $1-3,5-12$ \\
Native $\beta$ - sheet & $1626-1636$ & $1,4,6-11$ \\
Random coil & $1637-1655$ & $1,4,6,10,12,13$ \\
$\alpha-$ helix & $1656-1662$ & $1,4,6,10$ \\
B - turns & $1663-1696$ & $1,3,5,6,10,11,14$ \\
Parallel $\beta$ - sheet & $1697-1703$ & $1,10,11,15$
\end{tabular}

Table S 1: Peaks position for the assignment of the peaks after the Self-Fourier Deconvolution of the I Amide peak in the FTIR structural analysis. When the peak center is inside the bandwidth of a specific structure, that structure is assigned to the peak. 


\section{Weight Loss}

\begin{tabular}{c|cccccc} 
Source & Sum of Squares df Mean Square & F-value & p-value & \\
\hline Model & 3760.21 & 11 & 341.84 & 53.73 & $<0.0001$ & significant \\
A-Number of baths & 86.70 & 1 & 86.70 & 13.63 & 0.0007 & $* * *$ \\
B-Time & 126.23 & 1 & 126.23 & 19.84 & $<0.0001$ & $* * *$ \\
C-Temperature & 1411.00 & 1 & 1411.00 & 221.79 & $<0.0001$ & $* * *$ \\
D-Concentration & 1575.78 & 1 & 1575.78 & 247.70 & $<0.0001$ & $* * *$ \\
AC & 4.65 & 1 & 4.65 & 0.7309 & 0.3982 & \\
AD & 0.0079 & 1 & 0.0079 & 0.0012 & 0.9721 & \\
BC & 0.0395 & 1 & 0.0395 & 0.0062 & 0.9377 & \\
BD & 2.01 & 1 & 2.01 & 0.3152 & 0.5780 & $* * 0$ \\
CD & 402.11 & 1 & 402.11 & 63.21 & $<0.0001$ & $* * *$ \\
ACD & 26.34 & 1 & 26.34 & 4.14 & 0.0493 & $*$ \\
BCD & 125.34 & 1 & 125.34 & 19.70 & $<0.0001$ & $* * *$ \\
Residual & 229.02 & 36 & 6.36 & & & \\
\hline Lack of Fit & 12.69 & 4 & 3.17 & 0.4694 & 0.7577 & not significant \\
Pure Error & 216.33 & 32 & 6.76 & & & \\
Cor Total & 3989.23 & 47 & & & &
\end{tabular}

Table S 2: ANOVA table for the model of the weight loss. The significance level was assigned as follow: $p \leq 0.1$ (.), $p \leq 0.05(*), p \leq 0.01$ (**), $p \leq 0.001$ (***). All the first order terms, and several second and third order terms resulted to be significant. In order of importance the significant terms were: A, B, C, D, $C D, B C D$ and $A C D$. It should be noticed that all the other terms were insert in the model to maintain the overall hierarchy.

\begin{tabular}{cccc} 
Factor & Model coefficients & 95\% CI Low & 95\% CI High \\
\hline Intercept & 18.16 & 17.42 & 18.90 \\
A-Number of baths & 1.34 & 0.6056 & 2.08 \\
B-Time & 1.62 & 0.8834 & 2.36 \\
C-Temperature & 5.42 & 4.68 & 6.16 \\
D-Concentration & 5.73 & 4.99 & 6.47 \\
AC & -0.3113 & -1.05 & 0.4271 \\
AD & 0.0128 & -0.7255 & 0.7512 \\
BC & 0.0287 & -0.7097 & 0.7670 \\
BD & 0.2044 & -0.5339 & 0.9427 \\
CD & -2.89 & -3.63 & -2.16 \\
ACD & -0.7408 & -1.48 & -0.0025 \\
BCD & -1.62 & -2.35 & -0.8776 \\
\hline Transformation & None & &
\end{tabular}

Table S 3: Coefficients of the coded equations of the weight loss model and of the 95\% confidence intervals. In this case the factors are normalized in the range $[-1,1]$ to allow a direct comparison between the coefficients of the different terms. No transforming function was applied to the data. 


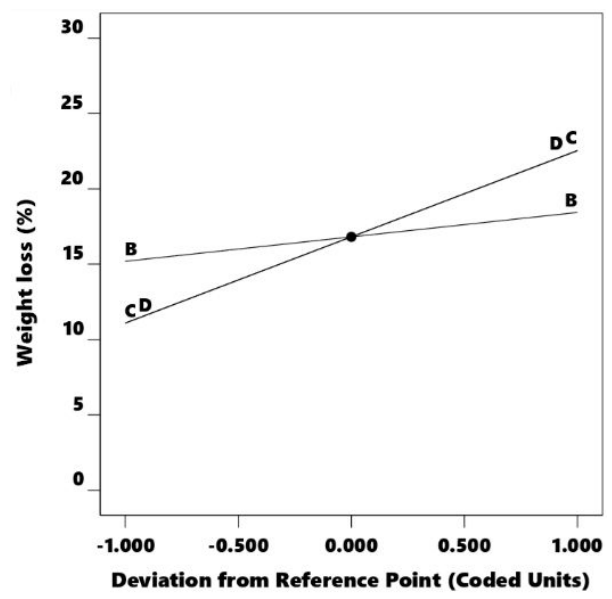

Figure S 3: Perturbation graph in coded terms of the weight loss. The variation of the terms $D$ and $C$ have a greater impact in terms of variation in the weight loss respect to a variation of the term $B$. In term of robustness, we can state that the process is more robust to a variation of $B$ if compared to the other terms. 


\section{Removed Sericin}

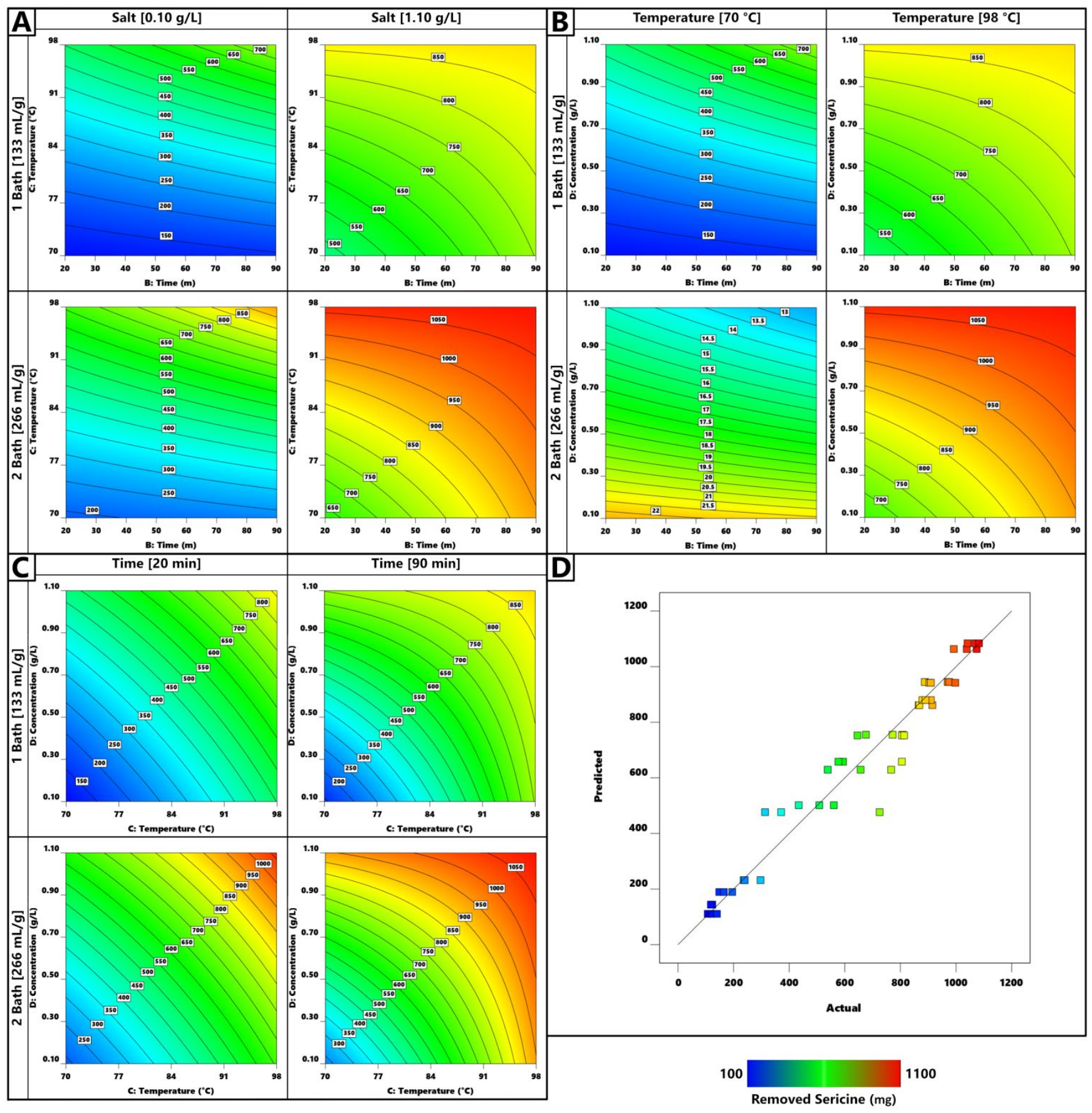

Figure S 4: Contour plots of the modelled amount of removed sericine (sericine present in the degumming bath). Due to the significance of all factors the model is 4 dimensional, so the contour plots are reported as follow: (A)removed sericine vs temperature and time, (B)removed sericine vs salt concentration and time, and (C) removed sericine vs salt concentration and temperature. In all these cases the number of baths is reported as an ordinal, and the missing variable in its extreme values. The actual versus predicted plot (D) shows that the model seems to fit better the data collected for the not successful degumming (in which there was only a small removal of the sericine) and the successful degumming (with a large amount or sericine solubilized) the data in between results to be scattered around the diagonal. 


\begin{tabular}{c|cccccc} 
Source & Sum of Squares & df & Mean Square & F-value & p-value & \\
\hline Model & 2556.45 & 8 & 319.56 & 120.56 & $<0.0001$ & significant \\
A-Number of baths & 127.76 & 1 & 127.76 & 48.20 & $<0.0001$ & $* * *$ \\
B-Time & 117.23 & 1 & 117.23 & 44.23 & $<0.0001$ & $* * *$ \\
C-Temperature & 1040.66 & 1 & 1040.66 & $392.61<0.0001$ & $* * *$ \\
D-Concentration & 973.49 & 1 & 973.49 & 367.27 & $<0.0001$ & $* * *$ \\
BC & 2.15 & 1 & 2.15 & 0.8098 & 0.3737 & \\
BD & 0.3131 & 1 & 0.3131 & 0.1181 & 0.7329 & \\
CD & 235.29 & 1 & 235.29 & 88.77 & $<0.0001$ & $* * *$ \\
BCD & 59.56 & 1 & 59.56 & 22.47 & $<0.0001$ & $* * *$ \\
Residual & 103.37 & 39 & 2.65 & & & \\
\hline Lack of Fit & 10.10 & 7 & 1.44 & 0.4949 & 0.8312 & not significant \\
Pure Error & 93.28 & 32 & 2.91 & & & \\
Cor Total & 2659.82 & 47 & & & &
\end{tabular}

Table S 4: ANOVA table for the model of the removed sericine. The significance level was assigned as follow: $p \leq 0.1$ (.), $p \leq 0.05$ (*), $p \leq 0.01$ (**), $p \leq 0.001$ (***). All the first order terms, and several second and third order terms resulted to be significant. In order of importance the significant terms were: A, B, $C, D, C D, B C D$, all the other terms were insert in the model to maintain the overall hierarchy. As could be expected, the significant terms are the same found in the analysis of the weight loss if we exclude the term ACD that in this case is not significant and in the previous one was slightly significant. This result confirms the correctness of the weight loss model.

\begin{tabular}{cccc} 
Factor & Model coefficients & 95\% CI Low & 95\% CI High \\
\hline Intercept & 24.15 & 23.67 & 24.62 \\
A-Number of baths & 1.63 & 1.16 & 2.11 \\
B-Time & 1.56 & 1.09 & 2.04 \\
C-Temperature & 4.66 & 4.18 & 5.13 \\
D-Concentration & 4.50 & 4.03 & 4.98 \\
BC & -0.2115 & -0.6868 & 0.2638 \\
BD & -0.0808 & -0.5561 & 0.3945 \\
CD & -2.21 & -2.69 & -1.74 \\
BCD & -1.11 & -1.59 & -0.6387 \\
\hline Transformation & Square root & &
\end{tabular}

Table S 5: Coefficients of the coded equations of the removed sericine model and of the 95\% confidence intervals. In this case the factors are normalized in the range [-1,1] to allow a direct comparison between the coefficients of the different terms. In this case the square root function was applied to the data in order to make the residues normal distributed.

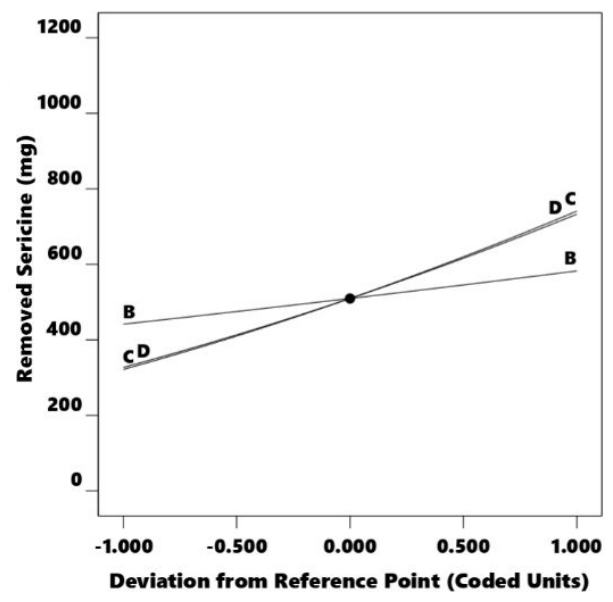

Figure S 5: Perturbation graph in coded terms of the removed sericine. As in the case of the weight loss the variation of the terms D and C have a greater impact in terms of sericine removal respect to a variation of the term B. In term of robustness, we can state that the process is more robust to a variation of $B$ if compared to the other terms. 
Fibers diameter

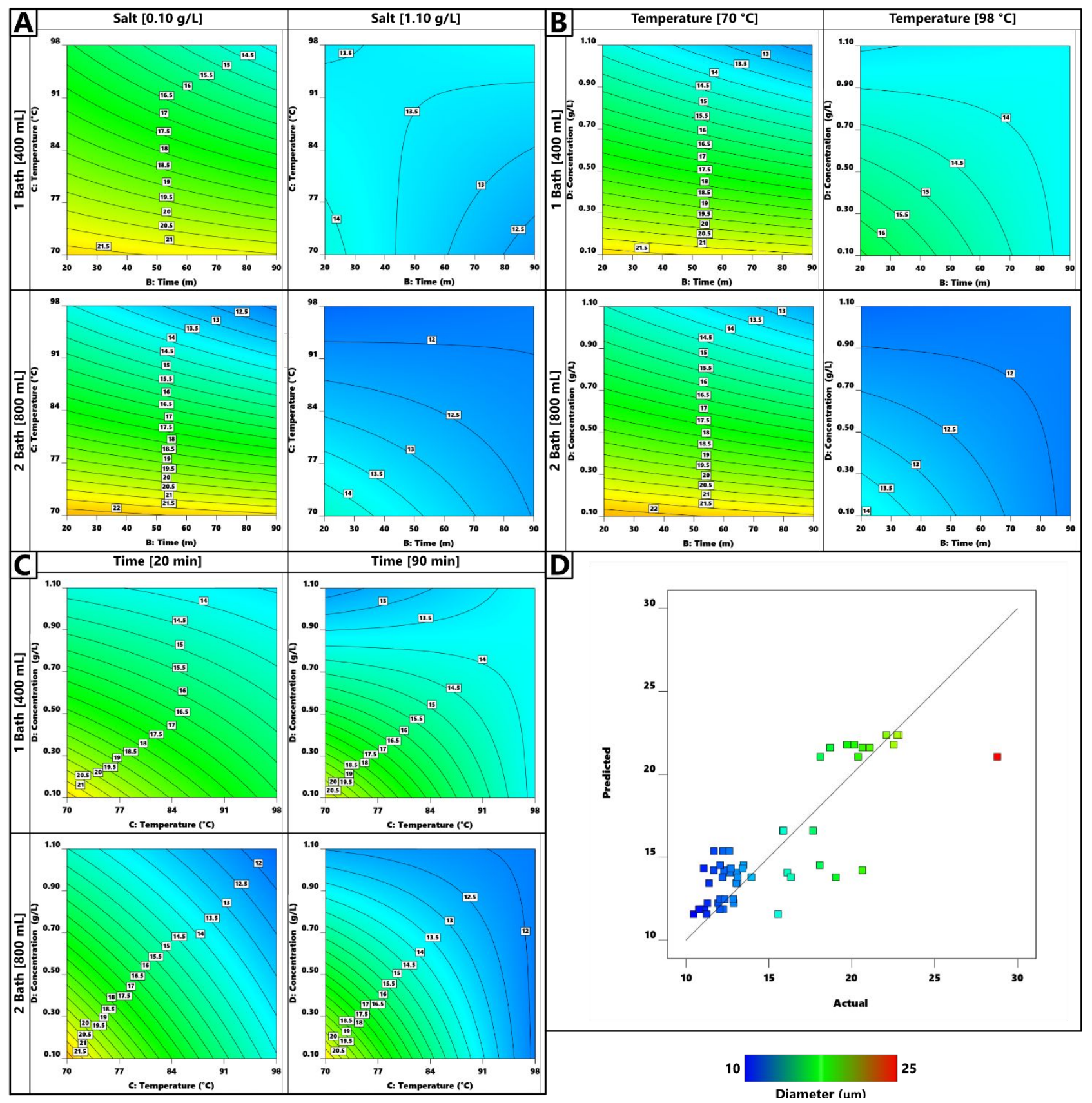

Figure S 6: Contour plots of the modelled fiber diameter (sericine present in the degumming bath). Due to the significance of all factors the model is 4 dimensional, so the contour plots are reported as follow: $(A)$ diameter vs temperature and time, $(B)$ diameter vs salt concentration and time, and (C) diameter $v s$ salt concentration and temperature. In all these cases the number of baths is reported as an ordinal, and the missing variable in its extreme values. The actual versus predicted plot (D) shows that the model seems to fit better the data collected for the successful degumming in which the diameter resulted to be lower. 


\begin{tabular}{c|cccccc} 
Source & Sum of Squares df & Mean Square & F-value & p-value & \\
\hline Model & 0.0100 & 9 & 0.0011 & 12.90 & $<0.0001$ & significant \\
A-Number of baths & 0.0003 & 1 & 0.0003 & 3.79 & 0.0582 &. \\
B-Time & 0.0004 & 1 & 0.0004 & 4.82 & 0.0335 & $*$ \\
C-Temperature & 0.0027 & 1 & 0.0027 & 31.56 & $<0.0001$ & $* * *$ \\
D-Concentration & 0.0041 & 1 & 0.0041 & 47.95 & $<0.0001$ & $* * *$ \\
AC & 0.0005 & 1 & 0.0005 & 6.23 & 0.0165 & $*$ \\
BC & $1.363 \mathrm{E}-06$ & 1 & $1.363 \mathrm{E}-06$ & 0.0159 & 0.9003 & \\
BD & 0.0000 & 1 & 0.0000 & 0.2823 & 0.5979 & $*$ \\
CD & 0.0014 & 1 & 0.0014 & 16.68 & 0.0002 & $* * *$ \\
BCD & 0.0004 & 1 & 0.0004 & 4.80 & 0.0340 & $*$ \\
Residual & 0.0037 & 43 & 0.0001 & & & \\
\hline Lack of Fit & 0.0003 & 7 & 0.0000 & 0.5191 & 0.8141 & not significant \\
Pure Error & 0.0034 & 36 & 0.0001 & & &
\end{tabular}

Table S 6: ANOVA table for the model of the fiber diameter. The significance level was assigned as follow: $p \leq 0.1$ (.), $p \leq 0.05$ (*), $p \leq 0.01$ (**), $p \leq 0.001$ (***). All the first order terms, and several second and third order terms resulted to be significant. In order of importance the significant terms were: $B$, $C$, $D, C D, B C D$, all the other terms were insert in the model to maintain the overall hierarchy. As could be expected, the significant terms are the same found in the analysis of the weight loss and the removed sericine if we exclude the term A (present in both the others model) and ACD (only present in the weight loss model). This result confirms the correctness of the previous two models.

\begin{tabular}{cccc} 
Factor & Model coefficients & 95\% CI Low 95\% CI High \\
Intercept & 0.0694 & 0.0667 & 0.0721 \\
A-Number of baths & 0.0025 & -0.0001 & 0.0050 \\
B-Time & 0.0029 & 0.0002 & 0.0056 \\
C-Temperature & 0.0075 & 0.0048 & 0.0102 \\
D-Concentration & 0.0093 & 0.0066 & 0.0120 \\
AC & 0.0033 & 0.0006 & 0.0060 \\
BC & -0.0002 & -0.0029 & 0.0025 \\
BD & -0.0007 & -0.0034 & 0.0020 \\
CD & -0.0055 & -0.0082 & -0.0028 \\
BCD & -0.0029 & -0.0056 & -0.0002 \\
\hline Transformation & Inverse & &
\end{tabular}

Table S 7: Coefficients of the coded equations of the fiber diameter model and of the $95 \%$ confidence intervals. In this case the factors are normalized in the range $[-1,1]$ to allow a direct comparison between the coefficients of the different terms. In this case the inverse transformation was applied to the data in order to make the residues normal distributed. 


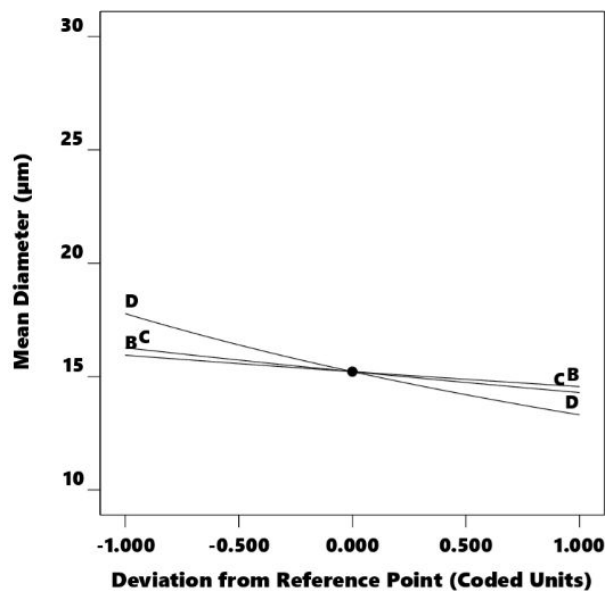

Figure S 7: Perturbation graph in coded terms of the removed sericine. As in the case of the weight loss and sericin removal the variation of the terms D and $C$ have a greater impact in terms of diameter variation respect to a variation of the term $B$. In term of robustness, we can state that the process is more robust to a variation of $B$ if compared to the other terms. 
Secondary structure
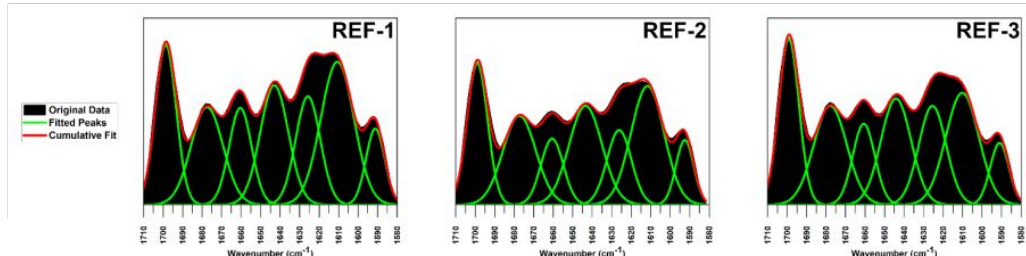

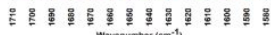
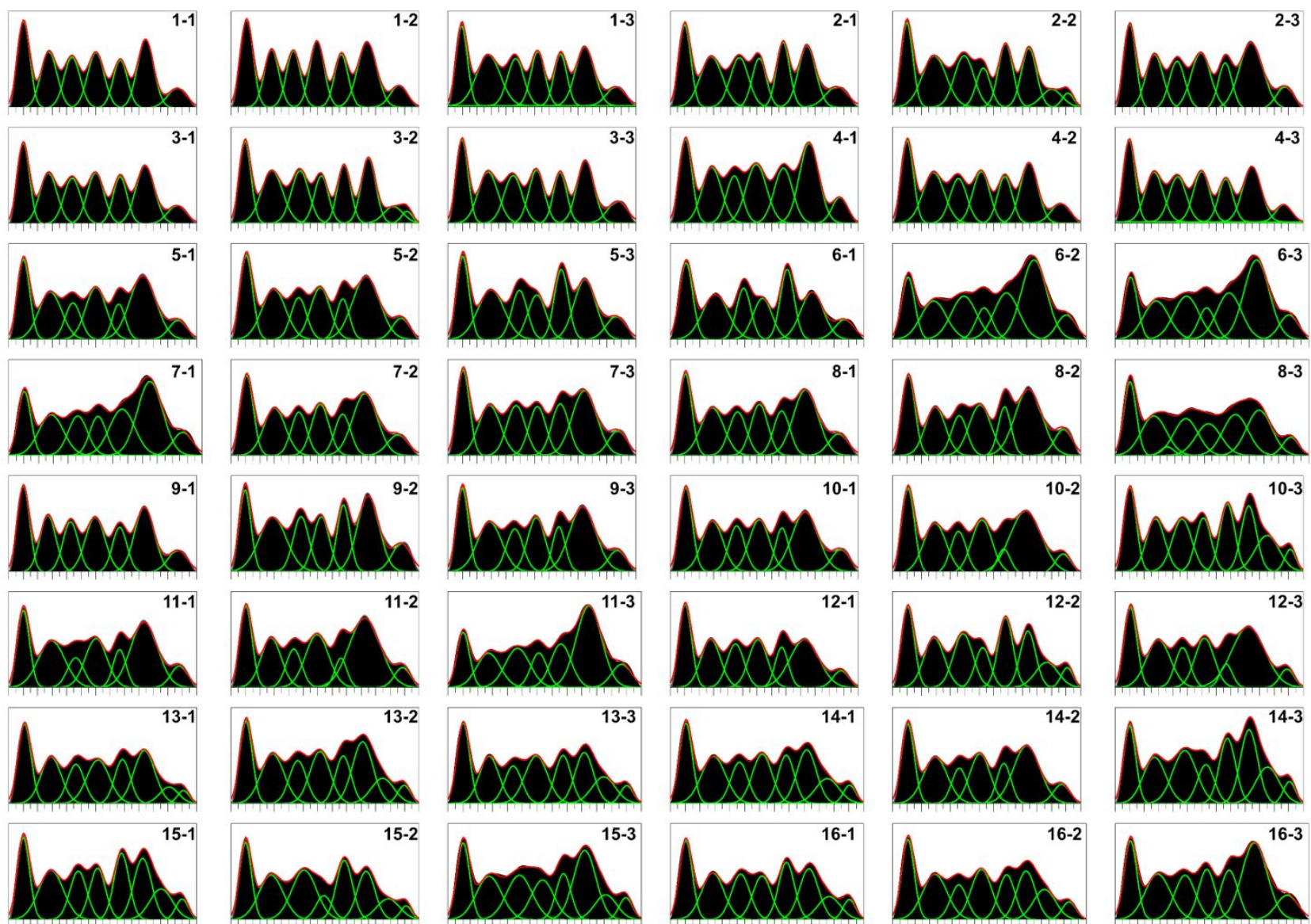

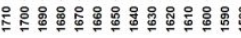

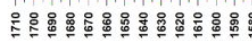

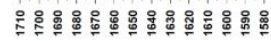

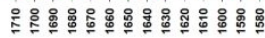

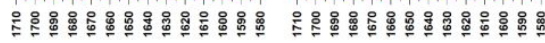

Figure S 8: Deconvolution and peak fitting of the 48 samples (3 trial for each of the 16 degumming procedure) and the three replicates of the reference procedure. The Amide I peak (1710-1580 cm-1$)$ was deconvolved by a Fourier Self -Deconvolution (FSD) the peaks were then identified by the second derivative. For each peak a secondary structure was assigned based on the assignment table (Table 4) and finally fitted with a Gaussian function. The area under each peak was taken as a measure of the amount of the specific secondary structure. It should be noticed that we didn't make any assumption in the number and position of the peaks before the deconvolution, and we always found 7 peaks for each sample, proving the robustness of this method. 


\begin{tabular}{c|cccccc} 
Source & Sum of Squares df Mean Square F-value p-value & \\
\hline Model & 150.55 & 9 & 16.73 & 3.29 & 0.0047 & significant \\
A-Number of baths & 0.3385 & 1 & 0.3385 & 0.0666 & 0.7978 & \\
B-Time & 0.1983 & 1 & 0.1983 & 0.0390 & 0.8445 & \\
C-Temperature & 9.86 & 1 & 9.86 & 1.94 & 0.1719 & \\
D-Concentration & 17.86 & 1 & 17.86 & 3.51 & 0.0686 &. \\
AB & 0.0268 & 1 & 0.0268 & 0.0053 & 0.9425 & \\
AD & 5.55 & 1 & 5.55 & 1.09 & 0.3030 & \\
BD & 8.20 & 1 & 8.20 & 1.61 & 0.2117 & $*$ \\
CD & 84.52 & 1 & 84.52 & 16.62 & 0.0002 & $* * *$ \\
ABD & 23.99 & 1 & 23.99 & 4.72 & 0.0362 & $*$ \\
Residual & 193.24 & 38 & 5.09 & & & \\
\hline Lack of Fit & 30.48 & 6 & 5.08 & 0.9987 & 0.4432 & not significant \\
Pure Error & 162.76 & 32 & 5.09 & & & \\
Cor Total & 343.79 & 47 & & & &
\end{tabular}

Table S 8: ANOVA table for the model of the $\beta$ structure percentage. The significance level was assigned as follow: $p \leq 0.1$ (.), $p \leq 0.05$ (*), $p \leq 0.01$ (**), $p \leq 0.001$ (***). Only one first order term, and one second and one third order terms resulted to be significant. In order of importance the significant terms were: $C D, A B D$ and $D$, all the other terms were insert in the model to maintain the overall hierarchy. Interestingly the amount of $\beta$-structures resulted to be mainly influenced by "high-order" terms that cannot be properly studied by the one-factor-at-time method.

\begin{tabular}{cccc} 
Factor & Model coefficient & 95\% CI Low & 95\% CI High \\
\hline Intercept & 52.73 & 52.07 & 53.39 \\
A-Number of baths & -0.0840 & -0.7429 & 0.5749 \\
B-Time & 0.0643 & -0.5946 & 0.7232 \\
C-Temperature & 0.4532 & -0.2057 & 1.11 \\
D-Concentration & -0.6100 & -1.27 & 0.0489 \\
AB & -0.0236 & -0.6825 & 0.6353 \\
AD & 0.3399 & -0.3190 & 0.9988 \\
BD & 0.4134 & -0.2455 & 1.07 \\
CD & -1.33 & -1.99 & -0.6680 \\
ABD & -0.7070 & -1.37 & -0.0481 \\
\hline Transformation & none & &
\end{tabular}

Table S 9: Coefficients of the coded equations of the percentage of $\beta$ structures model and of the $95 \%$ confidence intervals. The factors are normalized in the range $[-1,1]$ to allow a direct comparison between the coefficients of the different terms. In this case no transformation to the data was applied.

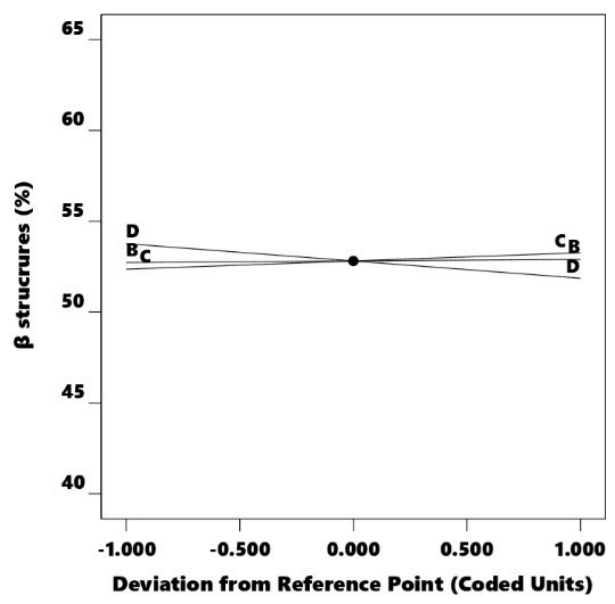

Figure S 9: Perturbation graph in coded terms of the percentage amount of $\beta$ structures. The variation of the terms $D$ and $C$ have a greater impact in terms of amount of $\beta$ structures respect to a variation of the term B. In term of robustness, we can state that the process is more robust to a variation of $B$ if compared to the other terms, even though only a slight there is only a slight difference between the three terms.

\section{Weight average molecular weight $\left(M_{w}\right)$}




\begin{tabular}{c|cccccc} 
Source & Sum of Squares & df & Mean Square & F-value & p-value & \\
\hline Model & 0.0000 & 9 & $3.399 \mathrm{E}-06$ & 40.61 & 0.0001 & significant \\
A-Number of baths & $2.300 \mathrm{E}-06$ & 1 & $2.300 \mathrm{E}-06$ & 27.48 & 0.0019 & $* *$ \\
B-Time & $3.690 \mathrm{E}-06$ & 1 & $3.690 \mathrm{E}-06$ & 44.09 & 0.0006 & $* * *$ \\
C-Temperature & 0.0000 & 1 & 0.0000 & 134.74 & $<0.0001$ & $* * *$ \\
D-Concentration & $7.338 \mathrm{E}-06$ & 1 & $7.338 \mathrm{E}-06$ & 87.67 & $<0.0001$ & $* * *$ \\
AB & $2.234 \mathrm{E}-06$ & 1 & $2.234 \mathrm{E}-06$ & 26.69 & 0.0021 & $* *$ \\
AC & $8.209 \mathrm{E}-08$ & 1 & $8.209 \mathrm{E}-08$ & 0.9808 & 0.3603 & \\
AD & $7.939 \mathrm{E}-08$ & 1 & $7.939 \mathrm{E}-08$ & 0.9486 & 0.3677 & \\
CD & $2.799 \mathrm{E}-06$ & 1 & $2.799 \mathrm{E}-06$ & 33.44 & 0.0012 & $* *$ \\
ACD & $7.878 \mathrm{E}-07$ & 1 & $7.878 \mathrm{E}-07$ & 9.41 & 0.0220 & $*$ \\
\hline Residual & $5.022 \mathrm{E}-07$ & 6 & $8.370 \mathrm{E}-08$ & & & \\
Cor Total & 0.0000 & 15 & & & &
\end{tabular}

Table S 10: ANOVA table for the model of $M_{w}$. The significance level was assigned as follow: $p \leq 0.1$ (.), $p \leq 0.05$ (*), $p \leq 0.01$ (**), $p \leq 0.001$ (***).All the first order terms were significant, as well as two second order terms and the one third order term. In order of importance the significant terms were: $C, D, B, A$, $C D, A B$ and $A C D$, all the other terms were insert in the model to maintain the overall hierarchy. As can be clearly observed $M_{w}$ is influenced by several factors and several mixed terms, difficult to observe with other methodologies.

\begin{tabular}{cccc} 
Factor & Coefficient Estimate & 95\% CI Low & 95\% CI High \\
\hline Intercept & 0.0044 & 0.0043 & 0.0046 \\
A-Number of baths & 0.0004 & 0.0002 & 0.0006 \\
B-Time & 0.0005 & 0.0003 & 0.0007 \\
C-Temperature & 0.0008 & 0.0007 & 0.0010 \\
D-Concentration & 0.0007 & 0.0005 & 0.0009 \\
AB & 0.0004 & 0.0002 & 0.0006 \\
AC & 0.0001 & -0.0001 & 0.0002 \\
AD & -0.0001 & -0.0002 & 0.0001 \\
CD & -0.0004 & -0.0006 & -0.0002 \\
ACD & 0.0002 & 0.0000 & 0.0004 \\
\hline Transformation & Inverse & & \\
\hline
\end{tabular}

Table S 11: Coefficients of the coded equations of the $M_{w}$ model and of the 95\% confidence intervals. The factors are normalized in the range [-1,1] to allow a direct comparison between the coefficients of the different terms. In this case no transformation to the data was applied.

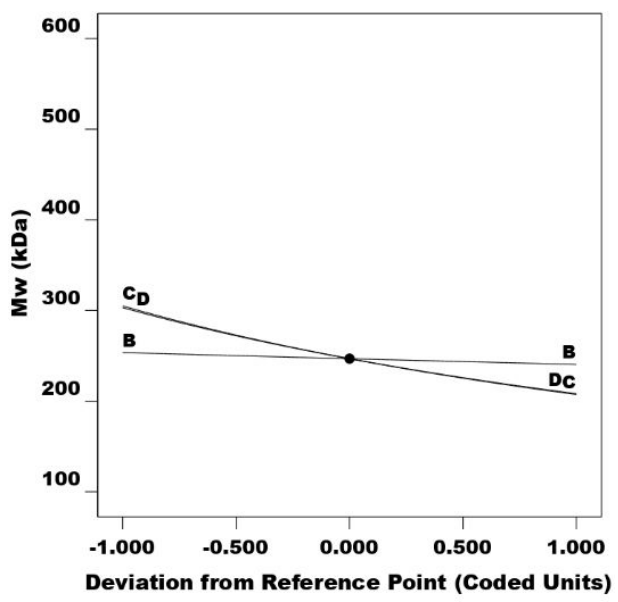

Figure S 10: Perturbation graph in coded terms of $M_{w}$. The variation of the terms $D$ and $C$ have a greater impact respect to a variation of the term $B$. In term of robustness, we can state that the process is more robust to a variation of $B$ if compared to the other terms, even though only a slight there is only a slight difference between the three terms. 
Number average molecular weight (Mn)

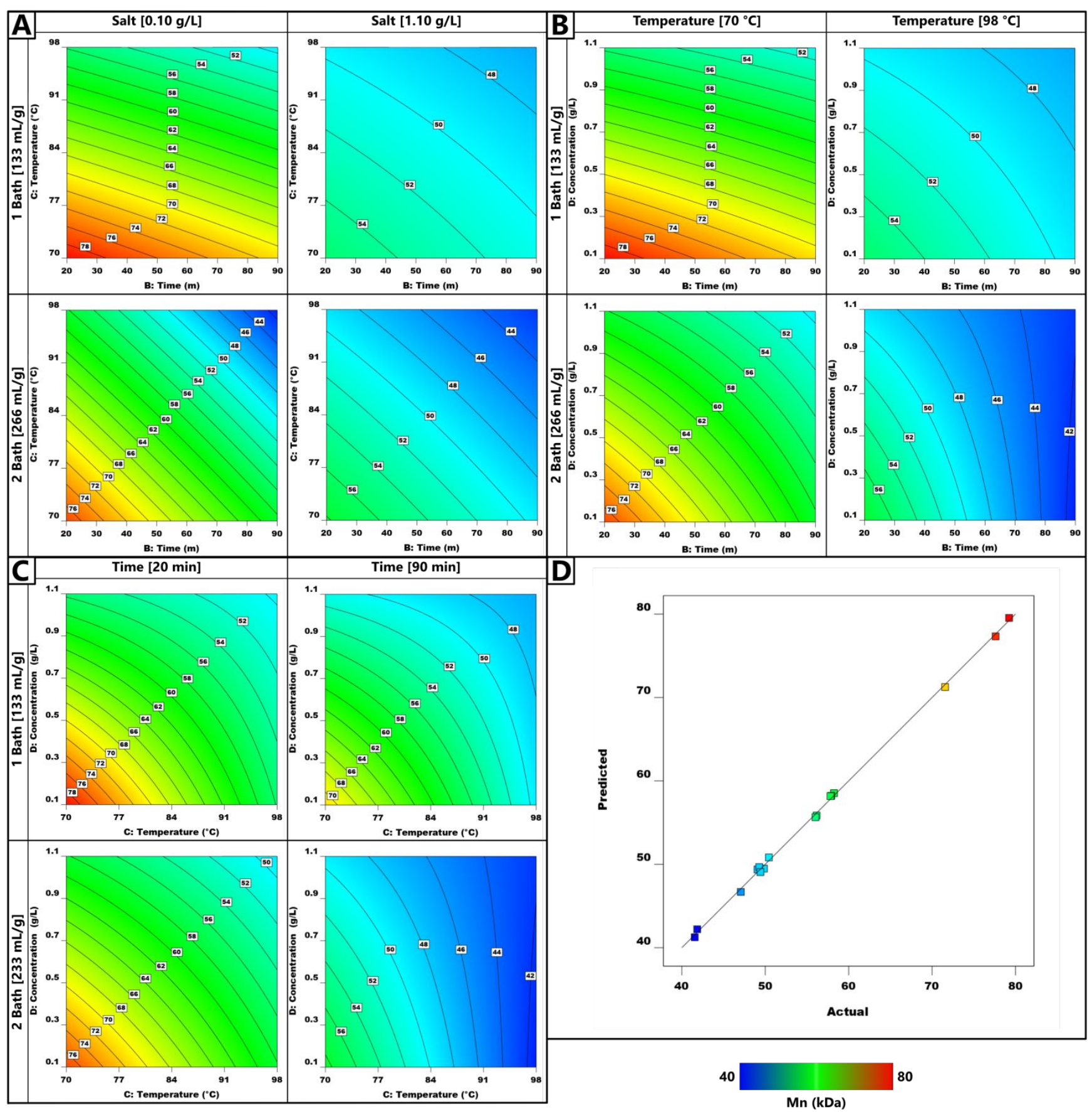

Figure S 11: Contour plots of the modelled $M_{n}$. Due to the significance of all factors the model is 4 dimensional, so the contour plots are reported as follow: (A) $M_{n} v s$ temperature and time, (B) $M_{n} v s$ salt concentration and time, and (C) $M_{n} v s$ salt concentration and temperature. In all these cases the number of baths is reported as an ordinal, and the missing variable in its extreme values. The actual versus predicted plot (D) shows that the model accurately fit the experimental data. 


\begin{tabular}{c|cccccc} 
Source & Sum of Squares & df & Mean Square & F-value & p-value & \\
\hline Model & 1952.77 & 12 & 162.73 & 264.65 & 0.0003 & significant \\
A-Number of baths & 37.76 & 1 & 37.76 & 61.41 & 0.0043 & $* *$ \\
B-Time & 340.82 & 1 & 340.82 & 554.27 & 0.0002 & $* * *$ \\
C-Temperature & 735.00 & 1 & 735.00 & $1195.33<0.0001$ & $* * *$ \\
D-Concentration & 501.79 & 1 & 501.79 & 816.07 & $<0.0001$ & $* * *$ \\
AB & 51.61 & 1 & 51.61 & 83.93 & 0.0027 & $* *$ \\
AC & 0.5230 & 1 & 0.5230 & 0.8505 & 0.4244 & \\
AD & 17.67 & 1 & 17.67 & 28.74 & 0.0127 & $*$ \\
BC & 3.38 & 1 & 3.38 & 5.49 & 0.1009 & \\
BD & 46.08 & 1 & 46.08 & 74.94 & 0.0032 & $* *$ \\
CD & 192.26 & 1 & 192.26 & 312.68 & 0.0004 & $* * *$ \\
ABD & 10.97 & 1 & 10.97 & 17.84 & 0.0243 & $*$ \\
ACD & 14.91 & 1 & 14.91 & 24.25 & 0.0161 & $* *$ \\
\hline Residual & 1.84 & 3 & 0.6149 & & & \\
Cor Total & 1954.61 & 15 & & & &
\end{tabular}

Table S 12: ANOVA table for the model of $M_{n}$. The significance level was assigned as follow: $p \leq 0.1$ (.), $p \leq 0.05$ (*), $p \leq 0.01$ (**), $p \leq 0.001$ (***).All the first order terms were significant, as well as four second order terms and the two third order term. In order of importance the significant terms were: $C, D, B$, $C D, A B, B D, A, A C D, A D$ and $A B D$ all the other terms were insert in the model to maintain the overall hierarchy. $M_{n}$ is influenced by several factors and several mixed terms, difficult to observe with other methodologies.

\begin{tabular}{cccc} 
Factor & Coefficient Estimate & 95\% CI Low & 95\% CI High \\
\hline Intercept & 55.82 & 55.20 & 56.44 \\
A-Number of baths & -1.54 & -2.16 & -0.9123 \\
B-Time & -4.62 & -5.24 & -3.99 \\
C-Temperature & -6.78 & -7.40 & -6.15 \\
D-Concentration & -5.60 & -6.22 & -4.98 \\
AB & -1.80 & -2.42 & -1.17 \\
AC & 0.1808 & -0.4431 & 0.8047 \\
AD & 1.05 & 0.4271 & 1.67 \\
BC & 0.4594 & -0.1644 & 1.08 \\
BD & 1.70 & 1.07 & 2.32 \\
CD & 3.47 & 2.84 & 4.09 \\
ABD & 0.8280 & 0.2042 & 1.45 \\
ACD & -0.9653 & -1.59 & -0.3414 \\
\hline Transformation & None & &
\end{tabular}

Table S 13: Coefficients of the coded equations of the $M_{n}$ model and of the 95\% confidence intervals. The factors are normalized in the range [-1,1] to allow a direct comparison between the coefficients of the different terms. In this case no transformation to the data was applied.

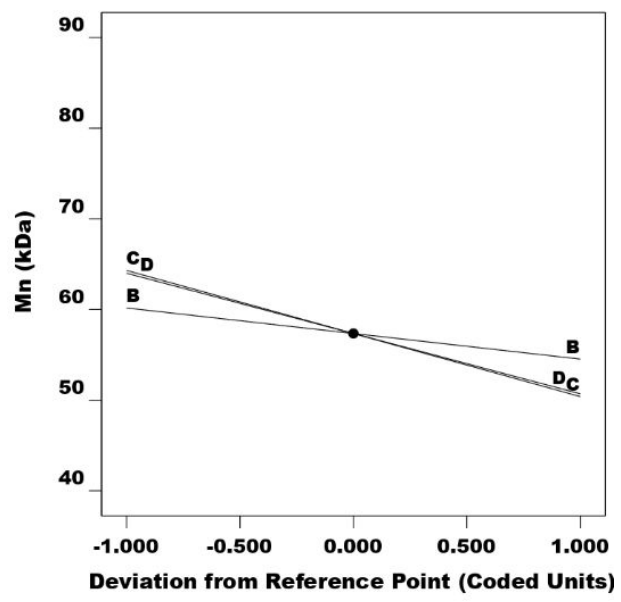

Figure S 12: Perturbation graph in coded terms of $M_{n}$. As could be expected this graph follows the trend of $M_{w}$, the variation of the terms $D$ and $C$ have a greater impact respect to a variation of the term B. In term of robustness, we can state that the process is more robust to a variation of $B$ if compared to the other terms, even though only a slight there is only a slight difference between the three terms. 
Peak molecular weight $\left(\mathbf{M}_{\mathrm{p}}\right)$

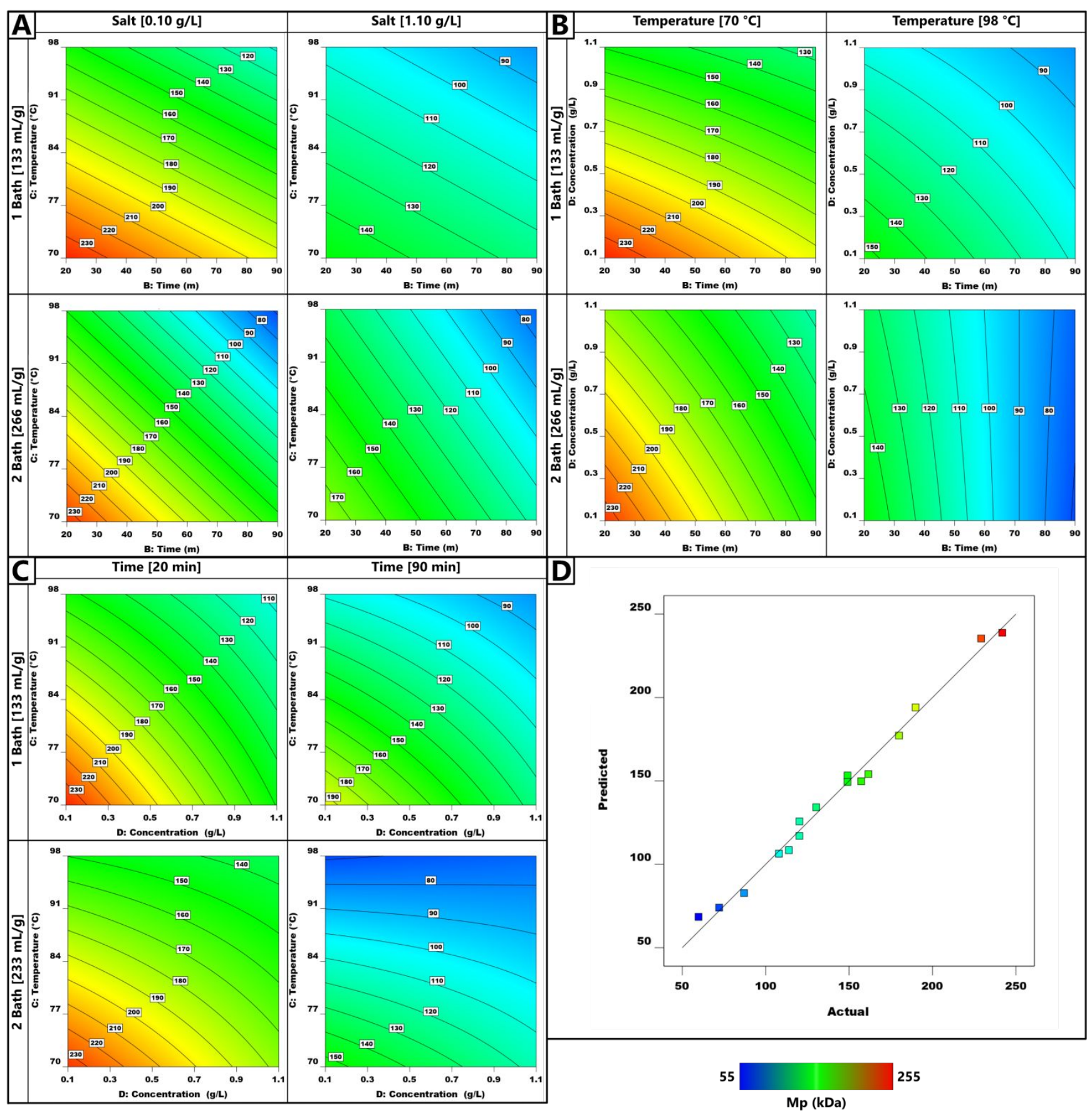

Figure S 13: Contour plots of the modelled $M_{p}$. Due to the significance of all factors the model is 4 dimensional, so the contour plots are reported as follow: (A) $M_{w} v s$ temperature and time, (B) $M_{p} v s$ salt concentration and time, and (C) $M_{p} v s$ salt concentration and temperature. In all these cases the number of baths is reported as an ordinal, and the missing variable in its extreme values. The actual versus predicted plot (D) shows that the model accurately fit the experimental data. 


\begin{tabular}{|c|c|c|c|c|c|c|}
\hline Source & Sum of Square & df & Mean Square & F-value & p-value & \\
\hline Model & 39305.97 & 8 & 4913.25 & 88.38 & $<0.0001$ & significant \\
\hline A-Number of baths & 147.52 & 1 & 147.52 & 2.65 & 0.1473 & \\
\hline B-Time & 11014.49 & 1 & 11014.49 & 198.13 & $<0.0001$ & $* * *$ \\
\hline C-Temperature & 16530.25 & 1 & 16530.25 & 297.34 & $<0.0001$ & $* * *$ \\
\hline D-Concentration & 7037.03 & 1 & 7037.03 & 126.58 & $<0.0001$ & $* * *$ \\
\hline $\mathrm{AB}$ & 1335.01 & 1 & 1335.01 & 24.01 & 0.0018 & $* *$ \\
\hline $\mathrm{AD}$ & 978.66 & 1 & 978.66 & 17.60 & 0.0041 & ** \\
\hline $\mathrm{BD}$ & 447.32 & 1 & 447.32 & 8.05 & 0.0252 & $*$ \\
\hline $\mathrm{CD}$ & 1815.70 & 1 & 1815.70 & 32.66 & 0.0007 & $* * *$ \\
\hline Residual & 389.15 & 7 & 55.59 & & & \\
\hline Cor Total & 39695.13 & 15 & & & & \\
\hline
\end{tabular}

Table S 14: ANOVA table for the model of $M_{p}$. The significance level was assigned as follow: $p \leq 0.1$ (.), $p \leq 0.05$ (*), $p \leq 0.01$ (**), $p \leq 0.001$ (***). All the first order terms were significant with the exclusion of the term A that was inserted to maintain the model hierarchy. Also, four second order resulted to be significant. In order of importance the significant terms were: $B, C, D, C D, A B, A D$ and $B D . M_{p}$ is influenced by several factors and several mixed terms, difficult to observe with other methodologies.

\begin{tabular}{cccc} 
Factor & Coefficient Estimate & 95\% CI Low & 95\% CI High \\
\hline Intercept & 141.82 & 137.41 & 146.22 \\
A-Number of baths & -3.04 & -7.44 & 1.37 \\
B-Time & -26.24 & -30.65 & -21.83 \\
C-Temperature & -32.14 & -36.55 & -27.73 \\
D-Concentration & -20.97 & -25.38 & -16.56 \\
AB & -9.13 & -13.54 & -4.73 \\
AD & 7.82 & 3.41 & 12.23 \\
BD & 5.29 & 0.8798 & 9.70 \\
CD & 10.65 & 6.25 & 15.06 \\
\hline Transformation & None & &
\end{tabular}

Table S 15: Coefficients of the coded equations of the $M_{n}$ model and of the 95\% confidence intervals. The factors are normalized in the range [-1,1] to allow a direct comparison between the coefficients of the different terms. In this case no transformation to the data was applied.

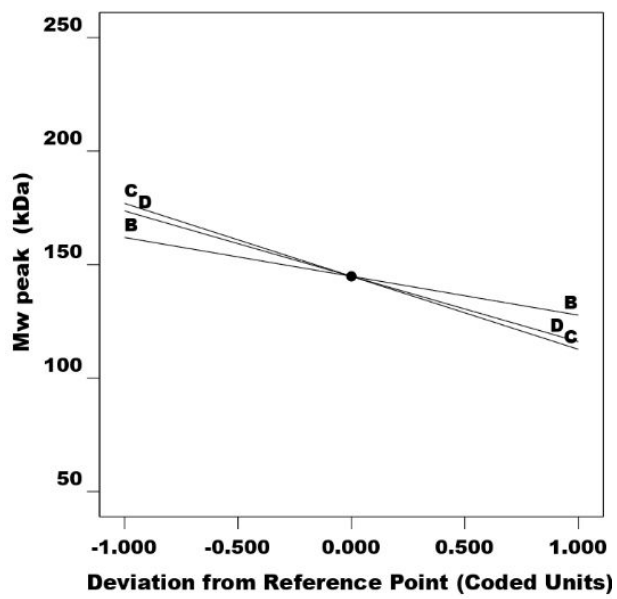

Figure S 14: Perturbation graph in coded terms of $M_{p}$. As could be expected this graph follows the trend of $M_{p}$, the variation of the terms $D$ and $C$ have a greater impact respect to a variation of the term B. In term of robustness, we can state that the process is more robust to a variation of $B$ if compared to the other terms, even though only a slight there is only a slight difference between the three terms. 


\section{Dispersion index (PDI)}

\begin{tabular}{ccccccc} 
Source & Sum of Squares df & Mean Square & F-value & p-value & \\
\hline Model & 0.0048 & 9 & 0.0005 & 16.19 & 0.0015 & significant \\
A-Number of baths & 0.0006 & 1 & 0.0006 & 17.22 & 0.0060 & $* *$ \\
B-Time & 0.0001 & 1 & 0.0001 & 4.49 & 0.0783 &. \\
C-Temperature & 0.0013 & 1 & 0.0013 & 40.86 & 0.0007 & $* * *$ \\
D-Concentration & 0.0012 & 1 & 0.0012 & 36.71 & 0.0009 & $* * *$ \\
AB & 0.0004 & 1 & 0.0004 & 12.79 & 0.0117 &. \\
AC & $6.744 \mathrm{E}-07$ & 1 & $6.744 \mathrm{E}-07$ & 0.0205 & 0.8908 & \\
AD & $4.409 \mathrm{E}-06$ & 1 & $4.409 \mathrm{E}-06$ & 0.1340 & 0.7268 & \\
CD & 0.0008 & 1 & 0.0008 & 23.93 & 0.0027 & $* *$ \\
ACD & 0.0003 & 1 & 0.0003 & 9.57 & 0.0213 & $*$ \\
\hline Residual & 0.0002 & 6 & 0.0000 & & & \\
Cor Total & 0.0050 & 15 & & & &
\end{tabular}

Table S 16: ANOVA table for the model of D. The significance level was assigned as follow: $p \leq 0.1$ (.), $p \leq 0.05$ (*), $p \leq 0.01$ (**), $p \leq 0.001$ (***). All the first order terms resulted to be significant. Also, two second order and one third order term were significant. In order of importance the significant terms were: $C, D, C D, A, A C D$ and $B . D$ is influenced by several factors and several mixed terms, difficult to observe with other methodologies.

\begin{tabular}{cccc} 
Factor & Coefficient Estimate & 95\% CI Low & 95\% CI High \\
\hline Intercept & 0.0563 & 0.0528 & 0.0598 \\
A-Number of baths & 0.0059 & 0.0024 & 0.0095 \\
B-Time & 0.0030 & -0.0005 & 0.0065 \\
C-Temperature & 0.0092 & 0.0057 & 0.0127 \\
D-Concentration & 0.0087 & 0.0052 & 0.0122 \\
AB & 0.0051 & 0.0016 & 0.0086 \\
AC & 0.0002 & -0.0033 & 0.0037 \\
AD & -0.0005 & -0.0040 & 0.0030 \\
CD & -0.0070 & -0.0105 & -0.0035 \\
ACD & 0.0044 & 0.0009 & 0.0079 \\
\hline Transformation & -Square & &
\end{tabular}

Table S 17: Coefficients of the coded equations of the D model and of the 95\% confidence intervals. The factors are normalized in the range [-1,1] to allow a direct comparison between the coefficients of the different terms. In this case no transformation to the data was applied.

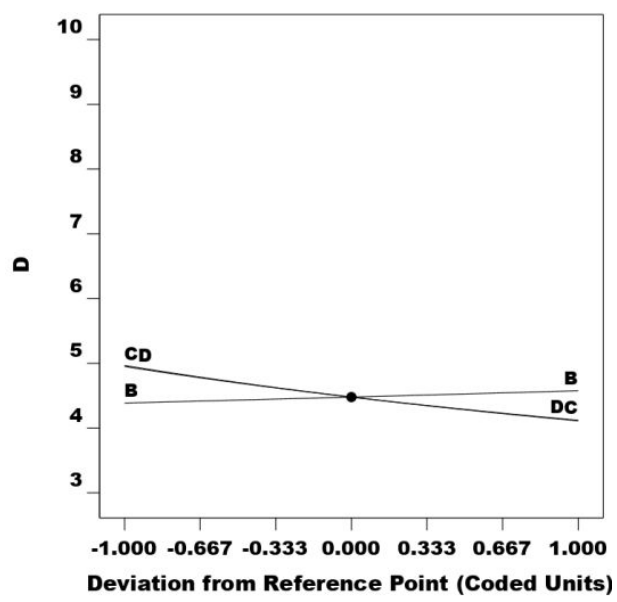

Figure S 15: Perturbation graph in coded terms of D. The variation of the terms $D$ and $C$ have a greater impact respect to a variation of the term B. In term of robustness, we can state that the process is more robust to a variation of $B$ if compared to the other terms, even though only a slight there is only a slight difference between the three terms. 


\section{Mechanical properties}

\section{Ultimate strength}

\begin{tabular}{c|cccccc} 
Source & Sum of Squares $\mathbf{d f}$ Mean Square & F-value & p-value & \\
\hline Model & $2.203 \mathrm{E}+05$ & 9 & 24482.07 & 5.29 & $<0.0001$ & significant \\
A-Number of baths & 12453.47 & 1 & 12453.47 & 2.69 & 0.1081 & \\
B-Time & 7198.83 & 1 & 7198.83 & 1.56 & 0.2190 & \\
C-Temperature & 4607.92 & 1 & 4607.92 & 0.9961 & 0.3238 & \\
D-Concentration & 95941.93 & 1 & 95941.93 & 20.74 & $<0.0001$ & $* * *$ \\
AC & 18274.41 & 1 & 18274.41 & 3.95 & 0.0532 &. \\
BC & 99.56 & 1 & 99.56 & 0.0215 & 0.8841 & \\
BD & 11824.72 & 1 & 11824.72 & 2.56 & 0.1172 & $*$ \\
CD & 38634.74 & 1 & 38634.74 & 8.35 & 0.0060 & $* *$ \\
BCD & 31303.02 & 1 & 31303.02 & 6.77 & 0.0127 & $*$ \\
Residual & $1.989 \mathrm{E}+05$ & 43 & 4625.85 & & & \\
\hline Lack of Fit & 20007.26 & 7 & 2858.18 & 0.5751 & 0.7712 & not significant \\
Pure Error & $1.789 \mathrm{E}+05$ & 36 & 4969.57 & & & \\
Cor Total & $4.557 \mathrm{E}+05$ & 53 & & & &
\end{tabular}

Table S 18: ANOVA table for the model of the ultimate strength. The significance level was assigned as follow: $p \leq 0.1$ (.), $p \leq 0.05$ (*), $p \leq 0.01$ (**), $p \leq 0.001$ (***). Only one first order term, two second and one third order terms resulted to be significant. In order of importance the significant terms were: D, CD, $B C D$ and $A C$ all the other terms were insert in the model to maintain the overall hierarchy.

\begin{tabular}{cccc} 
Factor & Model coefficients & 95\% CI Low & 95\% CI High \\
\hline Intercept & 348.84 & 329.04 & 368.63 \\
A-Number of baths & 15.19 & -3.48 & 33.85 \\
B-Time & -12.25 & -32.04 & 7.55 \\
C-Temperature & 9.80 & -10.00 & 29.60 \\
D-Concentration & 44.71 & 24.91 & 64.51 \\
AC & 19.51 & -0.2857 & 39.31 \\
BC & -1.44 & -21.24 & 18.36 \\
BD & -15.70 & -35.49 & 4.10 \\
CD & -28.37 & -48.17 & -8.57 \\
BCD & -25.54 & -45.33 & -5.74 \\
\hline Transformations & none & &
\end{tabular}

Table S 19: Coefficients of the coded equations of the ultimate strength model and of the 95\% confidence intervals. The factors are normalized in the range $[-1,1]$ to allow a direct comparison between the coefficients of the different terms. In this case no transformation to the data was applied.

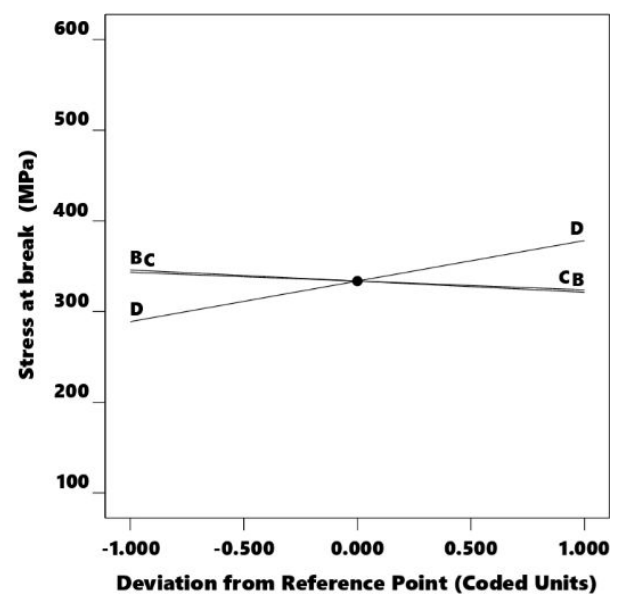

Figure S 16: Perturbation graph in coded terms of the stress at break. The variation of the terms D has a greater impact in terms of amount of $\beta$ structures respect to a variation of the other terms. In term of robustness, we can state that the process is more robust to a variation of $C$ and $B$ if compared to the term D. 


\section{Young's Modulus}

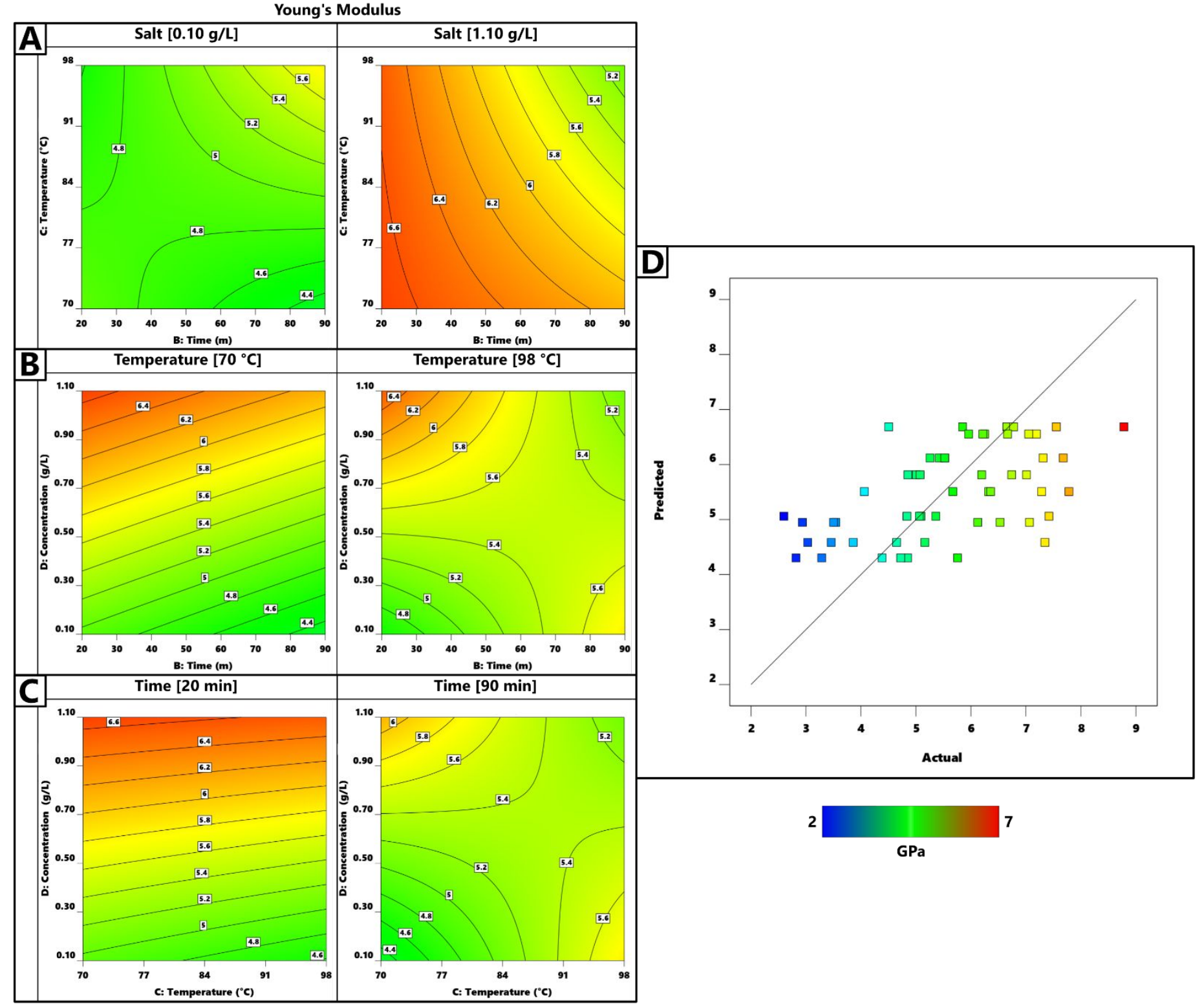

Figure S 17: Contour plot of the modelled Young's modulus (E) of the degummed silk fibers. In his case the number of baths term was not significant, all the other terms were instead significant, making the model dependent on 3 variables. In order of importance the significative terms were (ANOVA Table S20): $D$ (salt concentration), BCD (process time* process temperature* salt concentration), and BD (process time* salt concentration) The contour plots are reported as follows: $(\boldsymbol{A}) \mathrm{E}$ vs temperature and time, $(\boldsymbol{B}) \mathrm{E} v \mathrm{v}$ salt concentration and time, and $(\boldsymbol{C}) \mathrm{E} v \mathrm{~s}$ salt concentration and temperature. In all these cases the number of baths is reported as an ordinal, and the missing variable in its extreme values. As in the previous case, is extremely difficult to relate the Young's modulus to a specific property. The actual versus predicted plot (D) shows scattered point following a diagonal trend, this indicates a general agreement between the model and the collected values trends. 


\begin{tabular}{c|cccccc} 
Source & Sum of Squares df & Mean Square & F-value p-value & \\
\hline Model & 34.60 & 7 & 4.94 & 2.89 & 0.0139 & significant \\
B-Time & 1.64 & 1 & 1.64 & 0.9565 & 0.3333 & \\
C-Temperature & 0.0014 & 1 & 0.0014 & 0.0008 & 0.9772 & \\
D-Concentration & 17.07 & 1 & 17.07 & 9.98 & 0.0028 & $* *$ \\
BC & 0.6750 & 1 & 0.6750 & 0.3948 & 0.5330 & \\
BD & 5.24 & 1 & 5.24 & 3.06 & 0.0870 &. \\
CD & 4.09 & 1 & 4.09 & 2.39 & 0.1291 & \\
BCD & 5.90 & 1 & 5.90 & 3.45 & 0.0699 &. \\
Residual & 76.94 & 45 & 1.71 & & & \\
\hline Lack of Fit & 7.63 & 9 & 0.8479 & 0.4404 & 0.9038 & not significant \\
Pure Error & 69.31 & 36 & 1.93 & & & \\
Cor Total & 114.44 & 53 & & & &
\end{tabular}

Table S 20: ANOVA table for the model of the tensile modulus (E). The significance level was assigned as follow: $p \leq 0.1$ (.), $p \leq 0.05$ (*), $p \leq 0.01$ (**), $p \leq 0.001(* * *)$. Only one first order term, one second and one third order terms resulted to be significant. In order of importance the significant terms were: $D, B C D$, and $B D$ all the other terms were insert in the model to maintain the overall hierarchy.

\begin{tabular}{cccc} 
Factor & Model coefficients & 95\% CI Low & 95\% CI High \\
\hline Intercept & 5.51 & 5.13 & 5.89 \\
B-Time & -0.1846 & -0.5647 & 0.1956 \\
C-Temperature & -0.0054 & -0.3856 & 0.3747 \\
D-Concentration & 0.5963 & 0.2162 & 0.9764 \\
BC & 0.1186 & -0.2616 & 0.4987 \\
BD & -0.3303 & -0.7104 & 0.0499 \\
CD & -0.2918 & -0.6719 & 0.0884 \\
BCD & -0.3505 & -0.7306 & 0.0296 \\
\hline Treatments & none & &
\end{tabular}

Table S 21: Coefficients of the coded equations of the tensile modulus model and of the 95\% confidence intervals. The factors are normalized in the range $[-1,1]$ to allow a direct comparison between the coefficients of the different terms. In this case no transformation to the data was applied. 


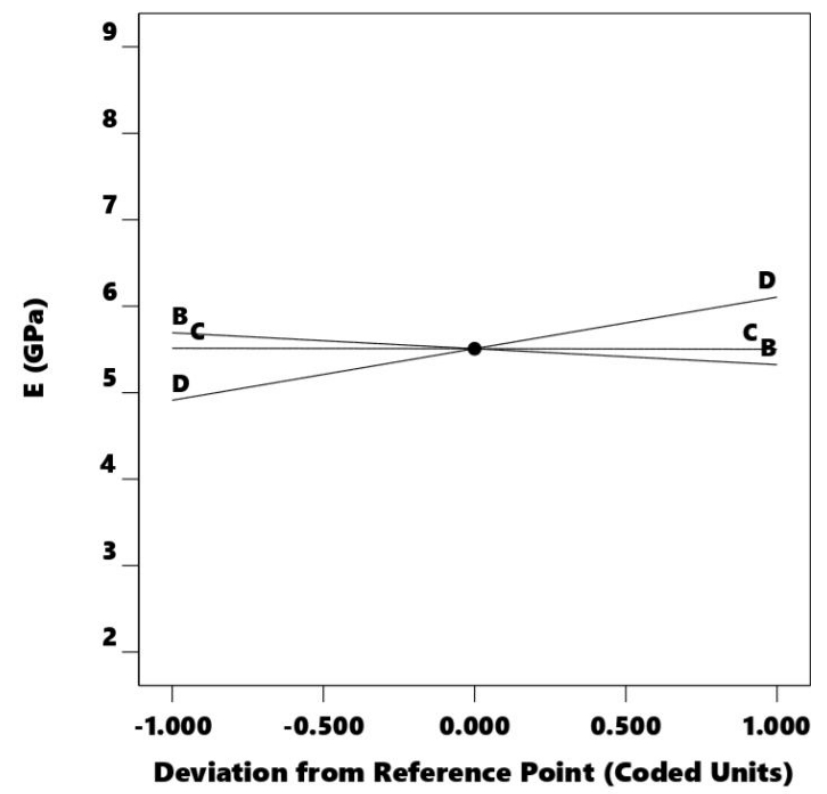

Figure S 18: Perturbation graph in coded terms of the tensile modulus. The variation of the terms D has a greater impact in terms of amount of $\beta$ structures respect to a variation of the other terms. In term of robustness, we can state that the process is more robust to a variation of $C$ and $B$ if compared to the term $D$ 


\section{Toughness}

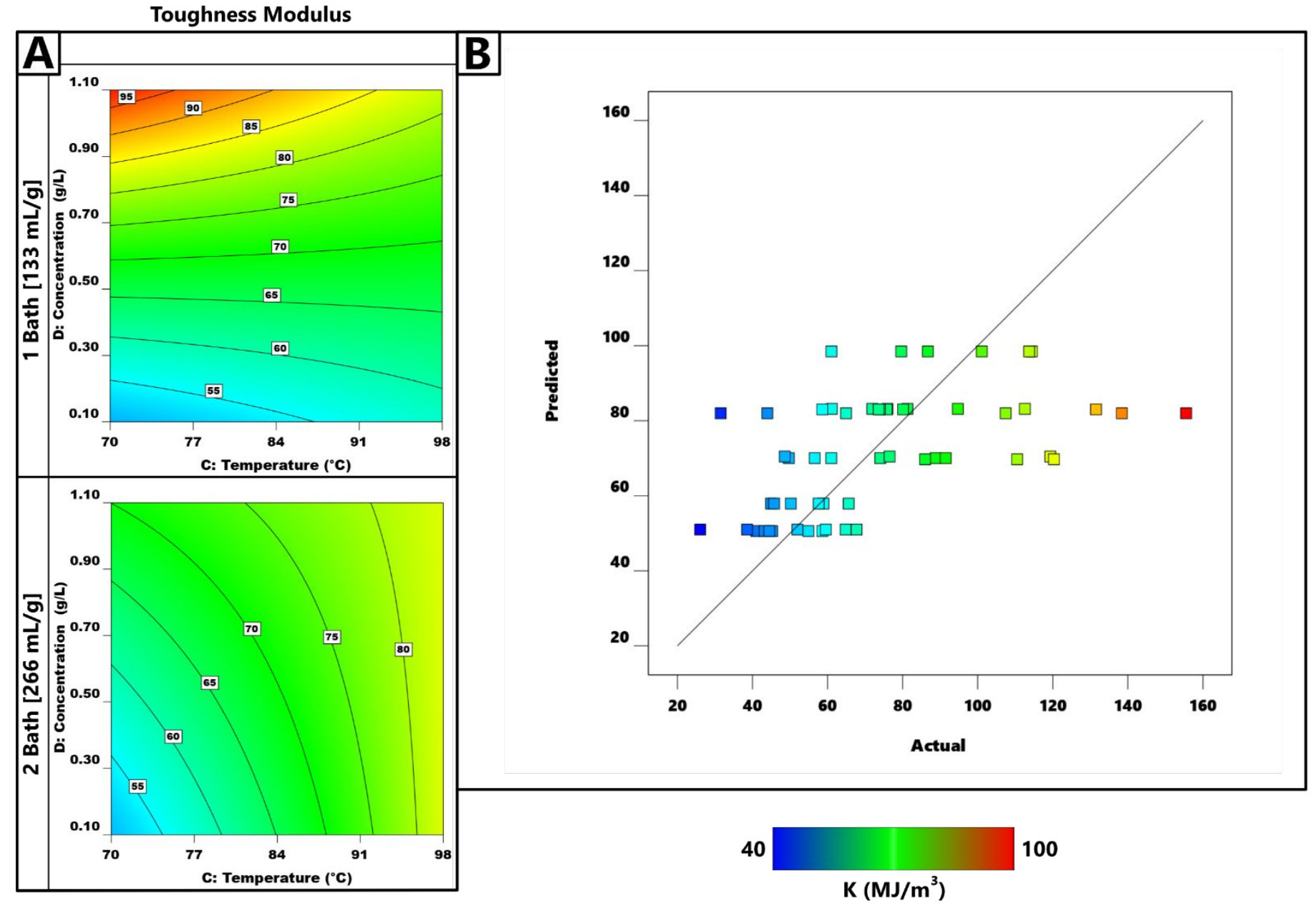

Figure S 19: Contour plot of the modelled toughness modulus (K) of the degummed silk fibres. The statistically significant terms (ANOVA Table S21), in order of importance, were: $D$ (salt concentration), $A C$ (number of baths*process temperature), AD (number of baths *salt concentration), and CD (process temperature* ${ }^{*}$ alt concentration). (A) $K$ vs salt concentration and temperature, the number of baths is reported as an ordinal, and the missing variable in its extreme values. The actual versus predicted plot $(\boldsymbol{B})$ shows scattered point following a diagonal trend, this indicates a general agreement between the model and the collected values trends. However, in this case the model was less precise than in all the previous cases because of the high variability on the obtained values. 


\begin{tabular}{c|cccccc} 
Source & Sum of Squares df & Mean Square & F-value & p-value & \\
\hline Model & 2.65 & 6 & 0.4417 & 4.21 & 0.0019 & significant \\
A-Number of baths & 0.0014 & 1 & 0.0014 & 0.0137 & 0.9074 & \\
C-Temperature & 0.2807 & 1 & 0.2807 & 2.68 & 0.1087 & \\
D-Concentration & 1.32 & 1 & 1.32 & 12.61 & 0.0009 & $* * *$ \\
AC & 0.3747 & 1 & 0.3747 & 3.57 & 0.0651 & . \\
AD & 0.3650 & 1 & 0.3650 & 3.48 & 0.0686 & . \\
CD & 0.3046 & 1 & 0.3046 & 2.90 & 0.0952 & . \\
Curvature & 0.4628 & 1 & 0.4628 & 4.41 & 0.0412 & $*$ \\
Residual & 4.83 & 46 & 0.1049 & & & \\
\hline Lack of Fit & 0.8647 & 10 & 0.0865 & 0.7856 & 0.6422 & not significant \\
Pure Error & 3.96 & 36 & 0.1101 & & & \\
Cor Total & 7.94 & 53 & & & &
\end{tabular}

Table S 22: ANOVA table for the model of the toughness. The significance level was assigned as follow: $p \leq 0.1$ (.), $p \leq 0.05$ (*), $p \leq 0.01$ (**), $p \leq 0.001$ (***). Only one first order term, one second and one third order terms resulted to be significant. In order of importance the significant terms were: D, AC, $A D$, and $C D$. All the other terms were insert in the model to maintain the overall hierarchy.

\begin{tabular}{cccc} 
Factor & Model coefficients & 95\% CI Low & 95\% CI High \\
\hline Intercept & 1.82 & 1.78 & 1.86 \\
A-Number of baths & 0.0226 & -0.0171 & 0.0623 \\
D-Concentration & 0.0583 & 0.0162 & 0.1005 \\
AD & -0.0395 & -0.0816 & 0.0027 \\
\hline Transformation & Logarithmic & &
\end{tabular}

Table S 23: Coefficients of the coded equations of the Young Modulus model and of the 95\% confidence intervals. The factors are normalized in the range $[-1,1]$ to allow a direct comparison between the coefficients of the different terms. In this case a logarithmic transformation was applied to the data to make the residues normally distributed.

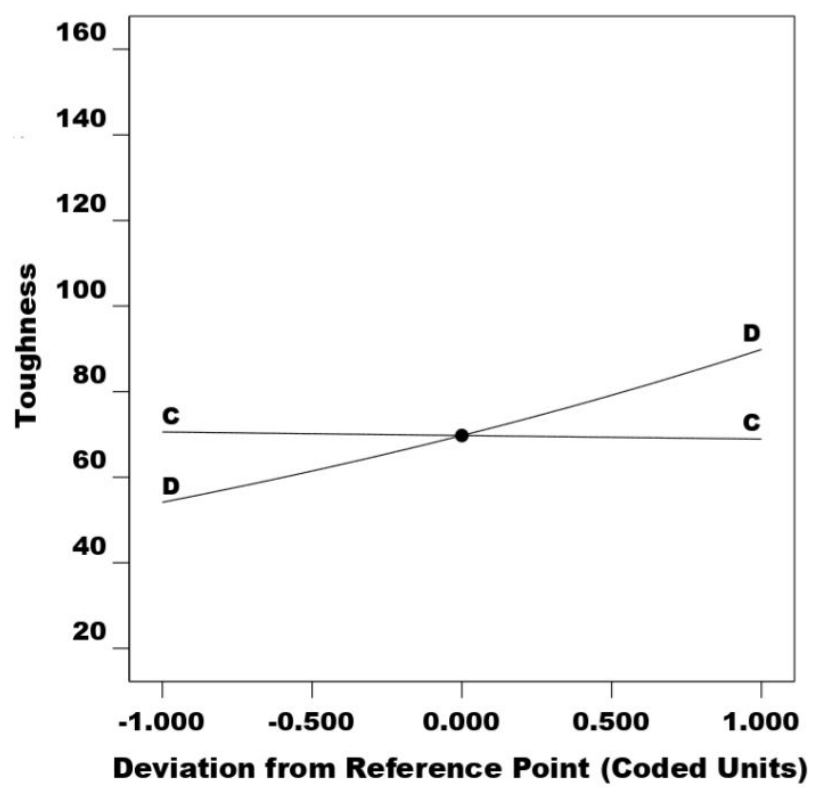

Figure S 20: Perturbation graph in coded terms of the toughness modulus. The variation of the terms $D$ has a greater impact in terms of K respect to a variation of the other term. In term of robustness, we can state that the process is more robust to a variation of $C$ if compared to the term $D$. 


\section{Mechanical properties vs molecular weight}

A

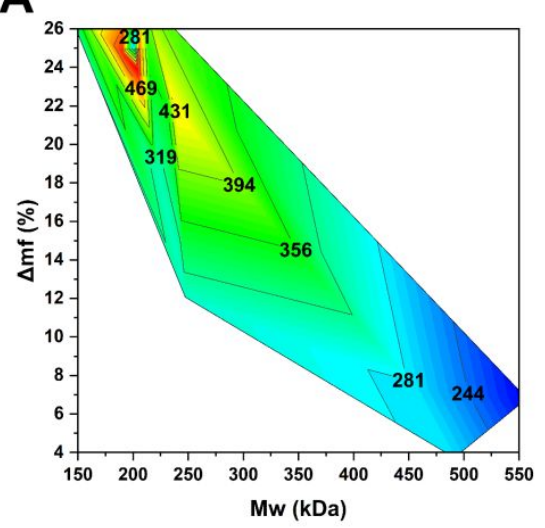

C

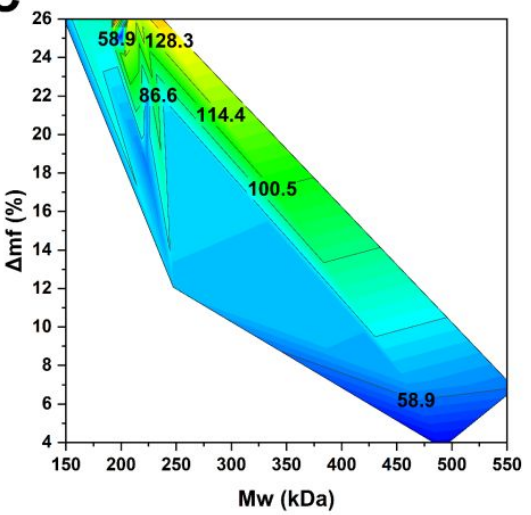

B
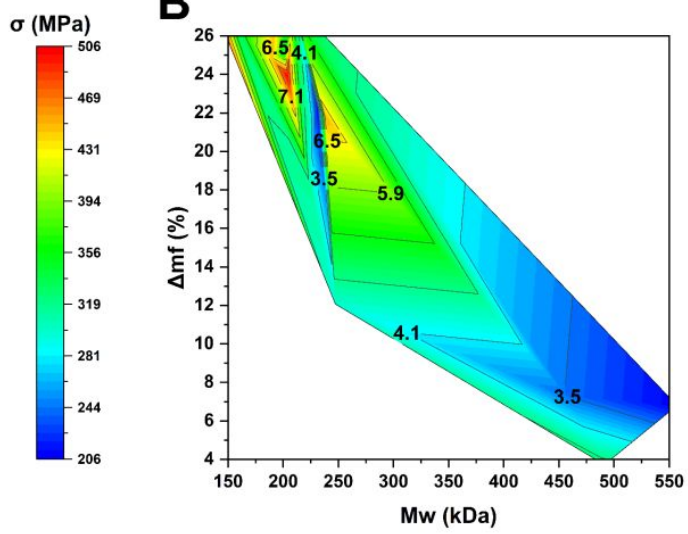

$\mathrm{E}(\mathrm{GPa})$

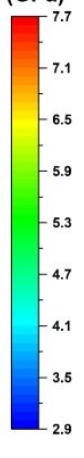

$\mathbf{D}_{20}$

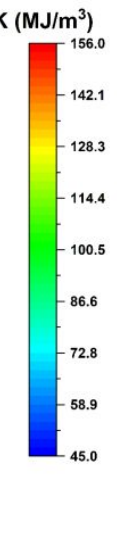

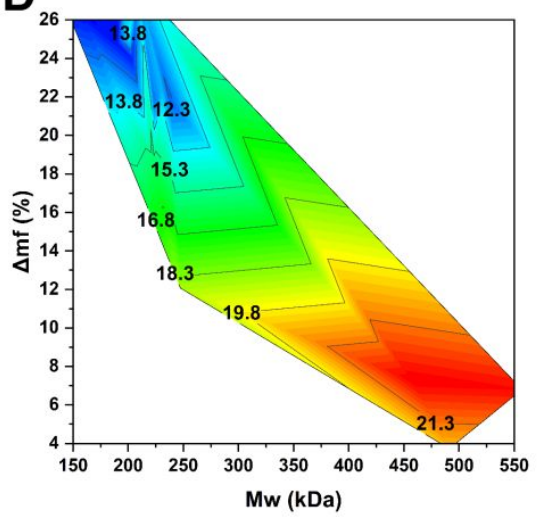

$\mathrm{d}(\mu \mathrm{m})$

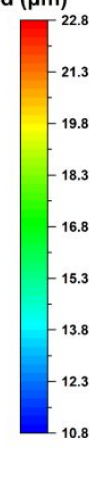

Figure S 21: Trends of (A) the ultimate strength, (B) the Young's modulus (D) the toughness index, and (D) the fibers mean diameter vs. the molecular weight and the weight loss. The blank area in the graph represents area not accessible with the current range choose for the process variables. These graphs have not to be intended as refined model punctual model (which is indeed the case of the empirical models that link the process variables to the material property) but as visualization of the general trend. In each of the reported graph is not possible to increase the mass loss (thus the amount of removed sericine) without decreasing the molecular weight. This explains why the decreasing of the molecular weight seems to improve the mechanical properties while instead is the decreasing of the sericine content that improv them. However, the two effects cannot be effectively separated if not extending the range of process variables or, by changing typology of degumming process. The conclusions on the mechanical property are further confirm from (D) in fact the lower part of the graph with the higher molecular weight results also in the higher diameter, implying fibers surrounded by sericin. 


\section{Empirical Models}

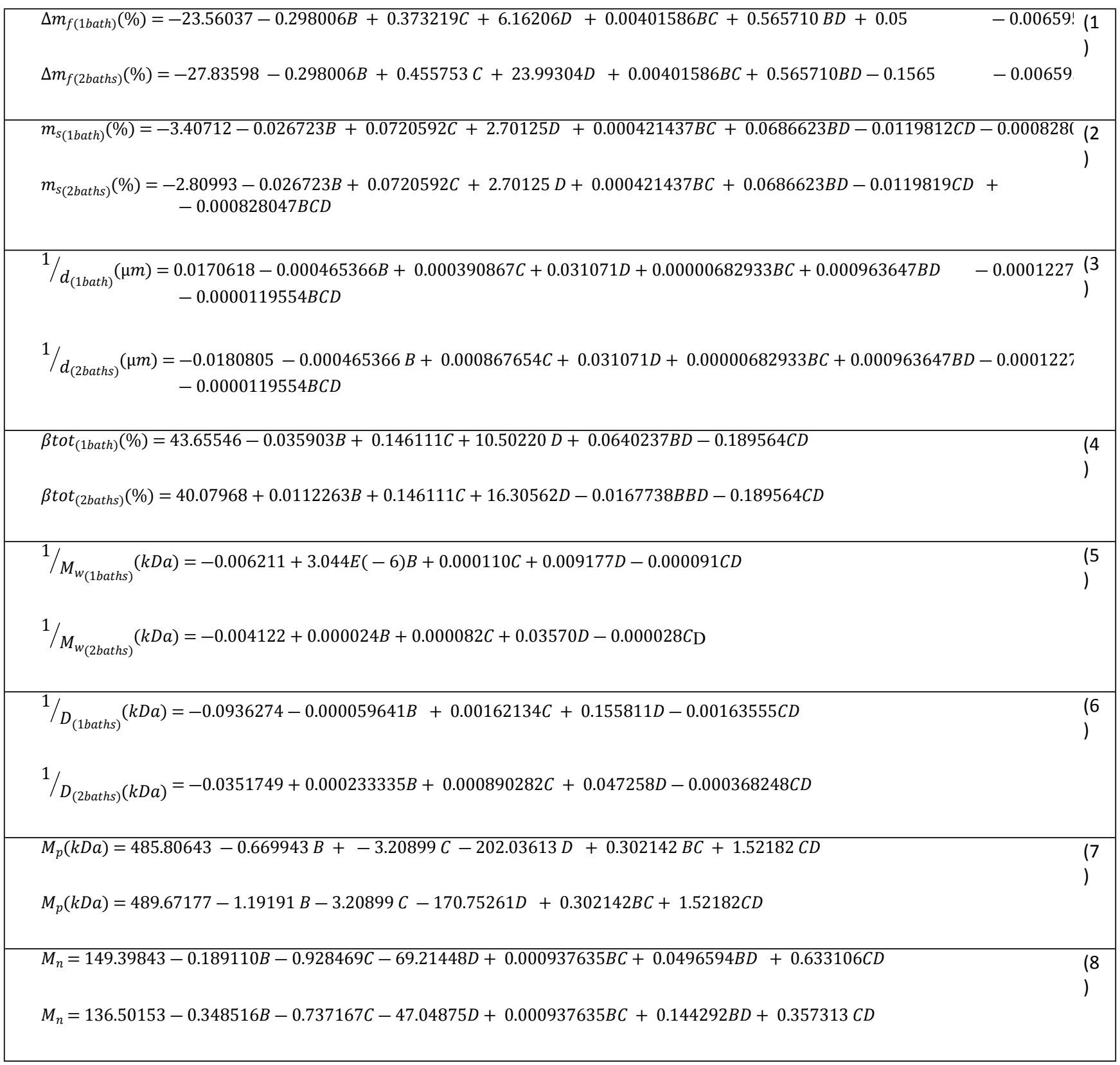




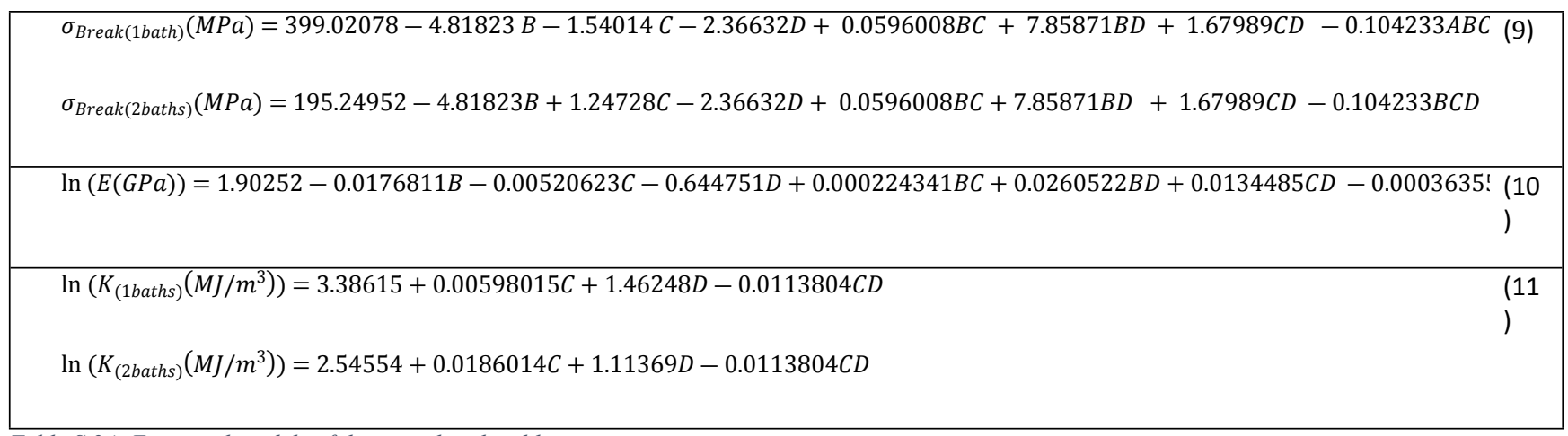

Table S 24: Empirical models of the considered yields. 


\section{References}

(1) Hu, X.; Kaplan, D.; Cebe, P. Determining Beta-Sheet Crystallinity in Fibrous Proteins by Thermal Analysis and Infrared Spectroscopy. Macromolecules 2006, 39 (18), 6161-6170. https://doi.org/10.1021/ma0610109.

(2) Jung, C. Insight into Protein Structure and Protein-Ligand Recognition by Fourier Transform Infrared Spectroscopy. Journal of Molecular Recognition. 2000. https://doi.org/10.1002/1099-1352(200011/12)13:6<325::AIDJMR507>3.0.CO;2-C.

(3) Mouro, C.; Jung, C.; Bondon, A.; Simonneaux, G. Comparative Fourier Transform Infrared Studies of the Secondary Structure and the CO Heme Ligand Environment in Cytochrome P-450cam and Cytochrome P-420cam. Biochemistry 1997. https://doi.org/10.1021/bi9700173.

(4) Teramoto, H.; Miyazawa, M. Molecular Orientation Behavior of Silk Sericin Film as Revealed by ATR Infrared Spectroscopy. Biomacromolecules 2005. https://doi.org/10.1021/bm0500547.

(5) Dong, A.; Huang, P.; Caughey, W. S. Protein Secondary Structures in Water from Second-Derivative Amide I Infrared Spectra. Biochemistry 1990. https://doi.org/10.1021/bi00465a022.

(6) Shimanovich, U.; Ruggeri, F. S.; De Genst, E.; Adamcik, J.; Barros, T. P.; Porter, D.; Müller, T.; Mezzenga, R.; Dobson, C. M.; Vollrath, F.; Holland, C.; Knowles, T. P. J. Silk Micrococoons for Protein Stabilisation and Molecular Encapsulation. Nat. Commun. 2017, 8 (May), 1-9. https://doi.org/10.1038/ncomms15902.

(7) Shivu, B.; Seshadri, S.; Li, J.; Oberg, K. A.; Uversky, V. N.; Fink, A. L. Distinct $\beta$-Sheet Structure in Protein Aggregates Determined by ATR-FTIR Spectroscopy. Biochemistry 2013. https://doi.org/10.1021/bi400625v.

(8) Ruggeri, F. S.; Longo, G.; Faggiano, S.; Lipiec, E.; Pastore, A.; Dietler, G. Infrared Nanospectroscopy Characterization of Oligomeric and Fibrillar Aggregates during Amyloid Formation. Nat. Commun. 2015. https://doi.org/10.1038/ncomms8831.

(9) Zandomeneghi, G.; Krebs, M. R. H.; McCammon, M. G.; Fändrich, M. FTIR Reveals Structural Differences between Native $\beta$-Sheet Proteins and Amyloid Fibrils. Protein Sci. 2009, 13 (12), 3314-3321. https://doi.org/10.1110/ps.041024904.

(10) Tretinnikov, O. N.; Tamada, Y. Influence of Casting Temperature on the Near-Surface Structure and Wettability of Cast Silk Fibroin Films. Langmuir 2001. https://doi.org/10.1021/1a010791y.

(11) Taddei, P.; Monti, P. Vibrational Infrared Conformational Studies of Model Peptides Representing the Semicrystalline Domains of Bombyx Mori Silk Fibroin. Biopolymers 2005. https://doi.org/10.1002/bip.20275.

(12) Goormaghtigh, E.; Cabiaux, V.; Ruysschaert, J. -M. Secondary Structure and Dosage of Soluble and Membrane Proteins by Attenuated Total Reflection Fourier-transform Infrared Spectroscopy on Hydrated Films. Eur. J. Biochem. 1990. https://doi.org/10.1111/j.1432-1033.1990.tb19354.x.

(13) Monti, P.; Freddi, G. Raman Spectroscopic Studies of Silk Fibroin from Bombyx Mori. J. Raman Spectrosc. 1998, 29 (January), 297-304. https://doi.org/10.1002/(SICI)1097-4555(199804)29:4<297::AID-JRS240>3.0.CO;2-G.

(14) Wilson, D.; Valluzzi, R.; Kaplan, D. Conformational Transitions Model Silk Peptides. Biophys. J. 2000. https://doi.org/10.1016/S0006-3495(00)76813-5.

(15) Chen, X.; Shao, Z.; Marinkovic, N. S.; Miller, L. M.; Zhou, P.; Chance, M. R. Conformation Transition Kinetics of Regenerated Bombyx Mori Silk Fibroin Membrane Monitored by Time-Resolved FTIR Spectroscopy. Biophys. Chem. 2001, 89 (1), 25-34. https://doi.org/10.1016/S0301-4622(00)00213-1. 